\title{
Disfunções cardiovasculares em traumatismo raquimedular
}

\author{
Liani Patrícia Andrade Santos', Eldon Bezerra da Silva Junior ${ }^{2}$, \\ Enaldo Vieira de $\mathrm{Melo}^{3}$, Carlos Umberto Pereira ${ }^{3}$
}

Universidade Federal de Sergipe (UFS), Aracaju, SE, Brasil e Serviço de Neurocirurgia do Hospital de Urgência de Sergipe, Aracaju, SE, Brasil.

\section{RESUMO}

Objetivo: Analisar frequência, tratamento e prognóstico das disfunções cardiovasculares em pacientes vítimas de traumatismo raquimedular (TRM). Métodos: Estudo longitudinal observacional, analítico e retrospectivo. Os dados foram coletados, retrospectivamente, de 49 pacientes da Unidade de Trauma, Hospital de Urgência de Sergipe, no período entre janeiro e dezembro de 2010. Resultados: A média de idade foi de $34,7 \pm 17,2$ anos, sendo 30 (61,2\%) pacientes entre 20 e 60 anos. O gênero masculino foi o mais acometido, sendo 44 (89,8\%). O tempo médio de internação foi de 22,5 dias \pm 21 . As principais causas de TRM foram: 18 quedas (37\%), 15 ferimentos por arma de fogo (31\%) e 12 acidentes automobilísticos (24\%). O TRM foi classificado em nível alto em 31 (63,3\%) casos e nível baixo em 18 (36,7\%). Durante a internação, cinco (10,2\%) evoluíram com bradicardia e 13 (26,5\%) apresentaram hipotensão arterial. Todos os casos de bradicardia tiveram hipotensão arterial associada. A média das PA sistólicas durante internação foi 109,1 $\pm 19,6 \mathrm{mmHg}$. A sobrevida global da amostra foi de $77,3 \%$ $\pm 7,5 \%$ em 20 dias. Os pacientes que apresentaram hipotensão arterial tiveram sobrevida menor em relação aos que não apresentaram. Nos pacientes com TRM nível alto, a sobrevida em 20 dias foi de $69,6 \% \pm 9,8 \%$ e nível baixo de $88,9 \% \pm 10,5 \%$. Conclusões: Bradicardia e hipotensão arterial são as disfunções cardiovasculares mais frequentes e estão associadas ao TRM nível alto, tendo pior prognóstico no tipo lesão completa.

\section{PALAVRAS-CHAVE}

Doenças cardiovasculares, traumatismos da medula espinal, sistema nervoso autônomo.

\begin{abstract}
Cardiovascular dysfunction in spinal cord trauma

Objective: Analyze frequency, treatment and prognosis of cardiovascular dysfunction in patients suffering from spinal cord trauma (TRM). Methods: Longitudinal observational, analytical and retrospective. Data were collected retrospectively from 49 patients of the Trauma Unit, Hospital Emergency Sergipe, in the period between January and December 2010. Results: Mean age was $34.7 \pm 17.2$, and $30(61.2 \%)$ patients were between 20 and 60 years. The male was involved in 44 (89.8\%). The mean hospital stay was 22.5 days \pm 21 . The main causes of TRM were 18 falls $(37 \%)$, gunshot wounds $15(31 \%)$ and motor vehicle accidents 12 (24\%). The TRM was rated highest level in 31 (63.3\%) cases and low 18 (36.7\%). During hospitalization, five (10.2\%) developed bradycardia and $13(26.5 \%)$ had hypotension. All cases had bradycardia associated with hypotension. The average systolic BP during hospitalization was $109.1 \pm 19.6 \mathrm{mmHg}$. The overall survival rate of the sample was $77.3 \% \pm 7.5 \%$ in 20 days. Patients who developed hypotension had lower survival than those who did not. In patients with high-level TRM survival at 20 days was $69.6 \% \pm 9.8 \%$ and low level of $88.9 \% \pm 10.5 \%$. Conclusions: Bradycardia and hypotension are the most common cardiovascular disorders and are associated with high TRM level, with poorer prognosis in complete lesion type.
\end{abstract}

\section{KEYWORDS}

Cardiovascular diseases, spinal cord injuries, autonomic nervous system.

1 Médica-residente do Serviço de Ginecologia e Obstetrícia do Instituto de Assistência Médica ao Servidor Público Estadual (IAMSPE), São Paulo, SP, Brasil.

2 Médico-residente do Serviço de Ortopedia do Hospital Municipal Dr. Carmino Caricchio, São Paulo, SP, Brasil.

3 Professores do Departamento de Medicina da Universidade Federal de Sergipe (UFS), Aracaju, SE, Brasil. 


\section{Introdução}

O traumatismo raquimedular (TRM) é um problema de saúde mundial, sendo um dos mais devastadores de todos os eventos traumáticos. A incidência anual de TRM é de 15 a 52,5 casos por milhão da população mundial, dos quais $80 \%$ correspondem a adultos jovens do sexo masculino ${ }^{1,2}$. A média de idade mais acometida é de 33,96 $\pm 13,56$ anos, com variância entre oito e 72 anos, sendo $39,1 \%$ dos casos entre 21 e 30 anos de idade. ${ }^{3,4}$

Estima-se que, na Alemanha, ocorram todos os anos 17 novos casos por milhão de habitantes e, nos Estados Unidos, essa cifra varia de 32 a 52 novos casos por milhão de habitantes. ${ }^{5,6}$ O Sistema Único de Saúde (SUS), em 2004, registrou 15.700 internações, com 505 óbitos decorrentes de TRM, com permanências hospitalares prolongadas, de alto custo e que envolvem equipes multidisciplinares. O número de pessoas tetraplégicas ou paraplégicas vem crescendo nas últimas décadas e hoje se estima no Brasil o total de seis a oito mil casos por ano. ${ }^{4}$

Alterações na função cardiovascular após TRM podem decorrer da lesão do sistema nervoso autônomo (SNA), que tem como função manter a estabilidade cardíaca. ${ }^{7}$ Ao reduzir a incidência da patologia cardiovascular, as taxas de morbimortalidade diminuem e a qualidade de vida dos pacientes pós-TRM melhoram..$^{8-11}$ O objetivo deste estudo é analisar frequência, tratamento e prognóstico das disfunções cardiovasculares em pacientes com TRM.

\section{Métodos}

O presente estudo foi longitudinal observacional e analítico com amostra não aleatória. Foram feitas coleta e análise retrospectiva dos dados de 49 pacientes internados durante o período de janeiro a dezembro de 2010, na Unidade de Emergência/Trauma do Hospital de Urgência de Sergipe (Aracaju/SE). Os dados foram coletados observando a ficha de admissão, anamnese e exame físico e neurológico dos pacientes com TRM. A amostra foi caracterizada quanto a gênero, idade, causa do trauma, nível da lesão medular e quadro clínico.

Foram incluídos no estudo pacientes de ambos os gêneros e todas as idades que tenham sido vítimas de TRM. Analisou-se se houve alteração da frequência cardíaca (FC) para valores iguais ou inferiores a 60 batimentos/minuto ou evolução para outras disfunções cardiovasculares, tais como hipotensão arterial (valores iguais ou inferiores a $90 / 60 \mathrm{mmHg}$ para as pressões sistólica e diastólica, respectivamente) e parada cardíaca, no período entre a admissão e alta hospitalar.
O prontuário foi analisado a cada 24 horas de internação até alta hospitalar. Foi considerado choque neurogênico a presença de bradicardia e hipotensão arterial. Após identificação e diagnóstico dos pacientes que apresentaram doença cardiovascular (DCV), o TRM foi classificado em nível alto (C1-T5) ou baixo (T6-L5) e essas DCV foram diferenciadas em cada grupo, descritas quanto a frequência, tratamento e prognóstico.

Foram excluídos deste estudo pacientes com história prévia de cardiopatia congênita ou adquirida e uso de drogas que predispõem à condição de disfunção cardiovascular. Foi utilizado o programa SPSS para análise estatística dos dados.

Este estudo foi aprovado pelo Comitê de Ética em Pesquisa da Universidade Federal de Sergipe (CAAE: 0275.0.107.000-11).

\section{Resultados}

Foram analisados 49 pacientes com TRM. A média de idade foi de $34,7 \pm 17,2$ anos, sendo $30(61,2 \%)$ pacientes entre 20 e 60 anos. $O$ gênero masculino foi o mais acometido, sendo $44(89,8 \%)$. O tempo médio de internação foi de 22,5 dias \pm 21 . As principais causas de TRM foram: 18 (37\%) quedas, 15 (31\%) ferimentos por arma de fogo e 12 (24\%) acidentes automobilísticos. A lesão foi cervical em 26 (53\%) casos, seguida pela lesão torácica em 15 (31\%) e lombar em 8 casos (16\%). O TRM foi classificado em nível alto em 31 (63,3\%) casos e nível baixo em 18 (36,7\%). Houve lesão medular completa em nove (18,4\%) pacientes. O quadro clínico foi déficit sensitivo em 33 (67,3\%), arreflexia profunda em 29 (59,2\%), paralisia flácida em $28(57,1 \%)$ e tetraplegia em $20(40,8 \%)$.

Na admissão, nove (18,4\%) apresentaram hipotensão arterial. No entanto, durante a internação, cinco $(10,2 \%)$ evoluíram com bradicardia e 13 (26,5\%) apresentaram hipotensão arterial (Figura 1). Todos os casos de bradicardia tiveram hipotensão arterial associada. A média da pressão arterial (PA) sistólica na admissão foi 123,6 $\pm 17 \mathrm{mmHg}$ e durante internação, $109,1 \pm 19,6 \mathrm{mmHg}$ (Figura 2). A probabilidade em função do tempo (dias) de ocorrência do evento bradicardia e/ou hipotensão arterial foi de 7,8\% em quatro dias, 9,9\% em 12 dias, $13,1 \% \mathrm{em}$ 13 dias, 16,4\% em 14 dias e 19,8\% em 15 dias (Figura 3).

Nos pacientes com lesão nível alto, durante internação, 11 (35,5\%) apresentaram hipotensão arterial e quatro (12,9\%), bradicardia. A média da PA sistólica foi $105,9 \pm 21,8 \mathrm{mmHg}$. Nesse grupo de pacientes, $44,4 \%$ dos que tinham lesão completa apresentaram bradicardia $(\mathrm{p}<0,003)$ e $100 \%$ dos que tiveram lesão completa evoluíram para hipotensão arterial, contra apenas 14,3\% daqueles com lesão incompleta $(\mathrm{p}<0,0001)$. 
Nos pacientes com lesão nível baixo, dois $(11,1 \%)$ apresentaram hipotensão arterial e $1(5,6 \%)$ evoluiu com bradicardia. A média da PA sistólica foi 114,7 $\pm 13,9 \mathrm{mmHg}$. Todos os pacientes tiveram lesão incompleta.

Vasopressores foram usados em nove $(18,4 \%)$ pacientes e a noradrenalina foi a droga de escolha em cinco $(55,5 \%)$ casos, seguida da atropina em três (33,3\%) e dopamina um $(11,2 \%)$. A evolução para parada cardiorrespiratória seguida de óbito ocorreu em 10 (20,4\%) casos. Desses, oito pacientes eram do grupo TRM nível alto, representando $25,8 \%$ das vítimas desse grupo e dois eram TRM nível baixo, representando $11,1 \%$ dos casos desse grupo.

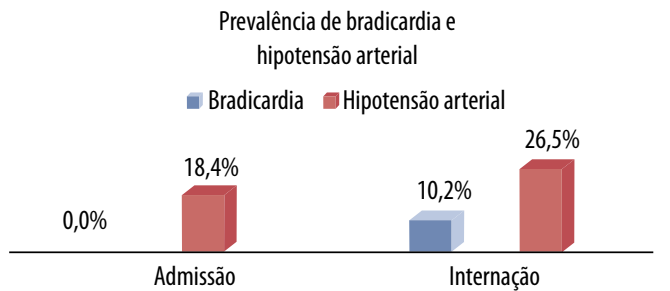

Figura 1 - Alteração da frequência cardíaca (FC) e pressão arterial (PA).

Média das PA sistólicas

- Admissão Internação

123,6

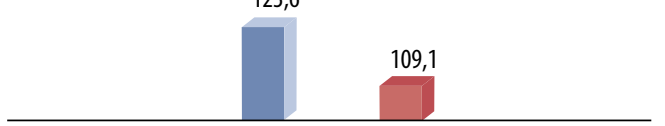

Figura 2 - Média das pressões arteriais sistólicas.

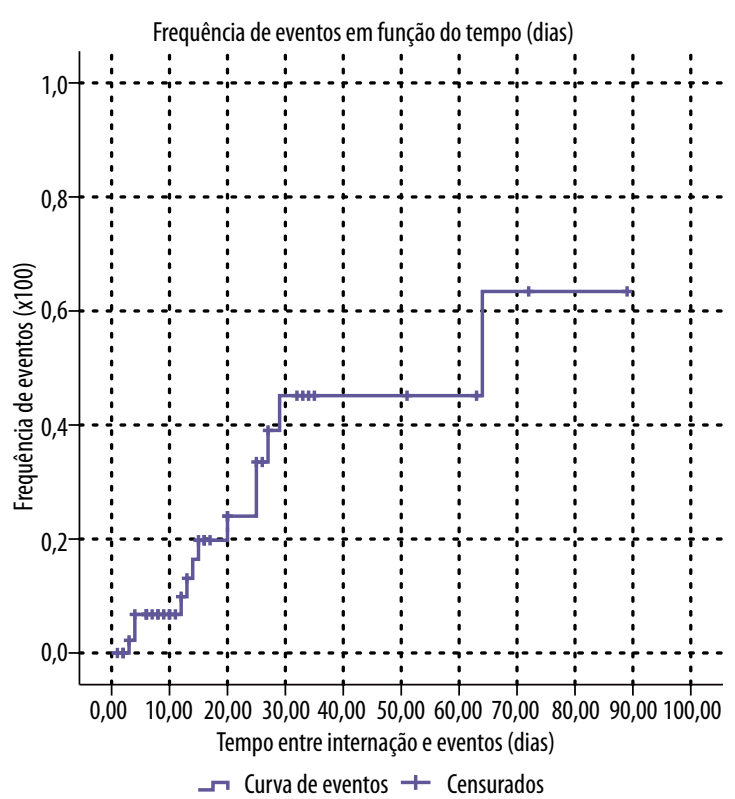

Figura 3 - Probabilidade de ocorrência do evento bradicardia e/ou hipotensão arterial em função do tempo de internamento (dias).
A sobrevida global da amostra foi de $77,3 \% \pm 7,5 \%$ em 20 dias (Figura 4). Os pacientes que apresentaram hipotensão arterial tiveram sobrevida menor em relação aos que não apresentaram (Figura 5). Nos pacientes com TRM nível alto, a sobrevida foi de $75 \% \pm 9 \%$ em 15 dias e $69,6 \% \pm 9,8 \%$ em 20 dias e nível baixo de $88,9 \% \pm 10,5 \%$ em 20 dias (Figura 6). A tabela 1 resume a caracterização da amostra em relação ao nível alto ou baixo do TRM.

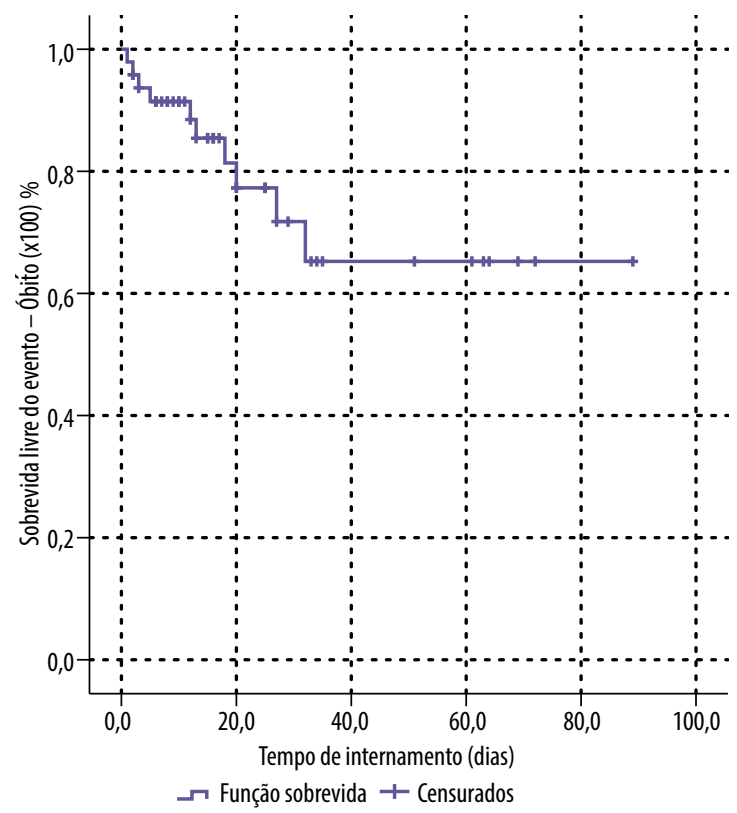

Figura 4 - Sobrevida global em função do tempo de internamento.

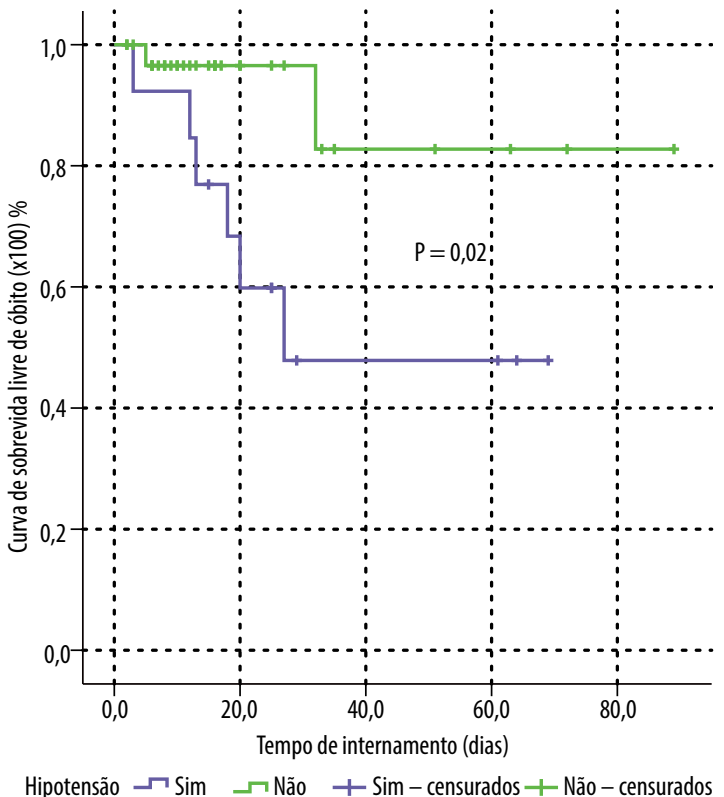

Figura 5 - Sobrevida global em relação aos pacientes que apresentaram ou não hipotensão arterial. 


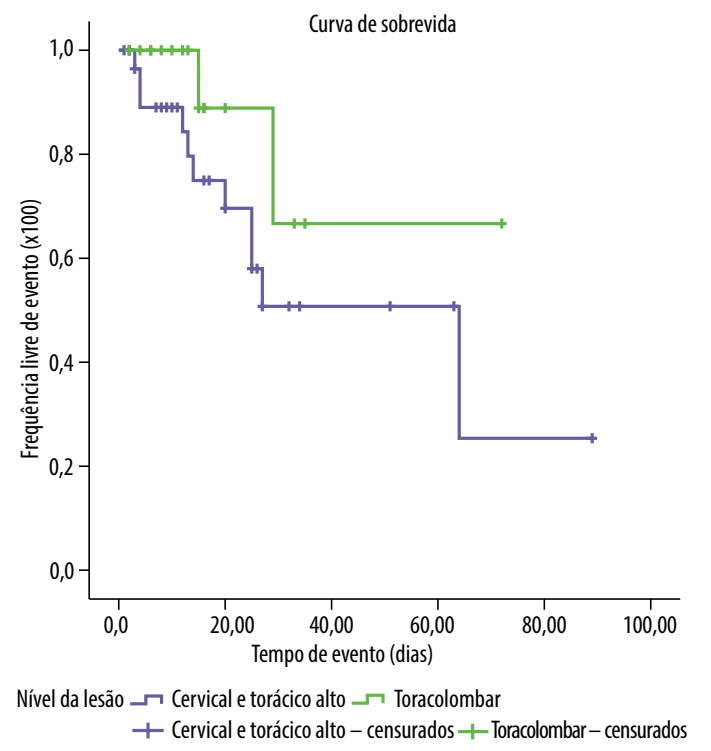

Figura 6 - Sobrevida em relação aos pacientes que tiveram nível alto ou baixo do TRM.

\begin{tabular}{|c|c|c|c|}
\hline \multicolumn{4}{|c|}{$\begin{array}{l}\text { Tabela } 1 \text { - Caracterização da amostra em } \\
\text { relação ao nível alto ou baixo do TRM }\end{array}$} \\
\hline \multicolumn{4}{|c|}{ Classificação do TRM n (\%) } \\
\hline Variáveis & $\begin{array}{c}\text { Alto } \\
(\mathrm{C} 1-\mathrm{T} 5) \\
\mathrm{N}=31\end{array}$ & $\begin{array}{c}\text { Baixo } \\
\text { (T6 - L5) } \\
\mathrm{N}=18\end{array}$ & \\
\hline \multicolumn{4}{|l|}{ Gênero } \\
\hline Masculino & $26(83,9 \%)$ & $18(100 \%)$ & $P=0,07$ \\
\hline Feminino & $5(16,1 \%)$ & 0 & \\
\hline \multicolumn{4}{|l|}{ Idade (anos) } \\
\hline Menores de 20 & $8(25,8 \%)$ & $3(16,7 \%)$ & $P=0,45$ \\
\hline Entre 20 e 60 & $18(58,1 \%)$ & $12(66,7 \%)$ & $\mathrm{P}=0,55$ \\
\hline Maiores de 60 & $5(16,1 \%)$ & $3(16,7 \%)$ & $P=0,96$ \\
\hline Média das idades (anos) & $33,9 \pm 17,1$ & $37,2 \pm 17,6$ & \\
\hline \multicolumn{4}{|l|}{ Causa de TRM } \\
\hline Quedas & $13(41,9 \%)$ & $5(27,8 \%)$ & $\mathrm{P}=0,32$ \\
\hline $\begin{array}{l}\text { Ferimentos por } \\
\text { arma de fogo }\end{array}$ & $8(25,8 \%)$ & $7(38,9 \%)$ & $\mathrm{P}=0,33$ \\
\hline Acidentes automobilísticos & $7(22,6 \%)$ & $5(27,8 \%)$ & $P=0,68$ \\
\hline $\begin{array}{l}\text { Ferimentos por } \\
\text { arma branca }\end{array}$ & $2(6,5 \%)$ & $1(5,6 \%)$ & $\mathrm{P}=0,89$ \\
\hline Outros & $1(3,1 \%)$ & 0 & \\
\hline \multicolumn{4}{|l|}{ Nível da lesão } \\
\hline Cervical & $26(83,9)$ & 0 & \\
\hline Torácico & $5(16,1 \%)$ & $10(55,6 \%)$ & $P=0,003$ \\
\hline Lombar & 0 & $8(44,4 \%)$ & \\
\hline Bradicardia & $4(12,9 \%)$ & $1(5,6 \%)$ & $\mathrm{P}=0,41$ \\
\hline Hipotensão & $11(35,5 \%)$ & $2(11,1 \%)$ & $P=0,06$ \\
\hline $\begin{array}{l}\text { Bradicardia }+ \\
\text { Hipotensão arterial } \\
\text { (choque neurogênico) }\end{array}$ & $4(12,9 \%)$ & $1(5,6 \%)$ & $\mathrm{P}=0,41$ \\
\hline Média das PAM & 82,4 & 86,4 & \\
\hline $\begin{array}{l}\text { Média das PA } \\
\text { sistólicas }(\mathbf{m m H g})\end{array}$ & $105,9 \pm 21,8$ & $114,7 \pm 13,9$ & \\
\hline Mortalidade & $8(25,8 \%)$ & $2(11,1 \%)$ & $\mathrm{P}=0,21$ \\
\hline Sobrevida em 20 dias & $69,6, \% \pm 9,8 \%$ & $88,9 \% \pm 10,5 \%$ & \\
\hline
\end{tabular}

\section{Discussão}

O TRM é uma condição patológica que provoca mudanças no estilo de vida do indivíduo por exigir adaptações às limitações físicas e às novas condições geradas. ${ }^{12}$ Em nossa casuística, a média de idade foi de $34,7 \pm 17,2$ anos, variância entre 16 e 64, sendo $61,2 \%$ da amostra entre 20 e 60 anos, mostrando consonância com a literatura médica. ${ }^{12-15}$ Segundo Lenehan et al., ${ }^{13}$ a média de idade das vítimas de TRM está entre 34,5 e 45,5 anos. No estudo de Ning et al., ${ }^{14}$ a média de idade correspondeu a $46 \pm 14,2$ anos, com variância entre 16 e 90 anos. Liu et al. ${ }^{15}$ relataram que $79,32 \%$ das vítimas de TRM estavam entre 20 e 60 anos de idade.

Neste estudo, a queda foi a principal causa do TRM, seguida de ferimentos por armas de fogo e acidentes automobilísticos, representando $90 \%$ da amostra. Ferimentos por arma de fogo (FAF), quedas de alturas ou por mergulho em águas rasas e acidentes automobilísticos têm sido as principais causas de TRM. ${ }^{4,14,16}$ Segundo Pickett et al., ${ }^{17}$ as principais causas de TRM são queda $(43,2 \%)$ e acidente de trânsito $(42,8 \%)$.

Neste estudo, o gênero masculino representou 89,8\% dos pacientes, proporção entre homens e mulheres de 8,8:1. Segundo Brito et al., ${ }^{4} 81,6 \%$ dos pacientes vítimas de TRM são do gênero masculino. A proporção de homens e mulheres é de 3:1 a 4:1. ${ }^{13,18}$ Segundo Ning et al., ${ }^{14}$ essa proporção chega a 5,63:1. Ocorre maior envolvimento do gênero masculino nas atividades profissionais que necessitam de trabalhos em alturas, nos acidentes automobilísticos e na violência por armas de fogo, fazendo com que o TRM seja mais prevalente na população masculina. ${ }^{19}$

Notou-se neste estudo que a lesão cervical foi a mais frequente, com 26 (53\%) casos, seguida pela lesão torácica com $15(30,6 \%)$ e lombar com oito $(16,3 \%)$ casos. Segundo Slucky e Eismont, ${ }^{20}$ dois terços das lesões por TRM estão localizadas no segmento cervical. Gonçalves et al. ${ }^{21} \mathrm{em}$ um estudo com 100 pacientes, observaram a prevalência do nível cervical, lombar e torácico, respectivamente. Ning et al. ${ }^{14}$ mostraram prevalência da lesão cervical em $71,5 \%$ dos casos, seguida por torácica em $13,3 \%$ e lombossacral em $15,1 \%$. No entanto, Brito et al. ${ }^{4}$ descreveram que os níveis mais frequentes são o torácico com $37,9 \%$, seguido do cervical e lombar, respectivamente com $26,4 \%$ e $25,4 \%$. Liu et al. ${ }^{15}$ relataram que fraturas e luxações ocorrem frequentemente nas vértebras cervicais e lombares, enquanto as lesões medulares ocorrem com frequência nos segmentos cervicais e torácicos.

As bradiarritmias ventriculares são as DCV mais frequentes, sendo a bradicardia reflexa um achado frequente na fase aguda. ${ }^{22} \mathrm{~A}$ hipotensão arterial é outra alteração cardiovascular que acomete $14,2 \%$ dos pacientes na fase aguda do TRM. A incidência de choque neurogênico em lesões cervicais é maior (19,3\%) quando comparada 
à lesão torácica e lombar, respectivamente $7 \%$ e $3 \%{ }^{23}$ Segundo Zipnick et al. ${ }^{24}$ o choque neurogênico está presente em 7\% dos pacientes com TRM. Este estudo revelou que, durante a internação, a prevalência de bradicardia nos 49 pacientes foi de cinco $(10,2 \%)$ casos e hipotensão arterial de 13 (26,5\%), sendo maiores quando comparadas às prevalências da admissão. Todos os casos que apresentaram bradicardia também evoluíram com hipotensão arterial. Dessa forma, observou-se que cinco $(10,2 \%)$ pacientes evoluíram com choque neurogênico. Naqueles com TRM nível alto, o choque neurogênico foi mais frequente, quatro $(12,9 \%)$ casos, quando comparado ao grupo de TRM nível baixo, 1 (5,6\%). A probabilidade do evento bradicardia e/ou hipotensão arterial foi maior quanto maior o tempo de internação. Dessa forma, foi demonstrado neste estudo que, em 4 dias, 93,2\% \pm $3,8 \%(\mathrm{~N}=41)$ estavam livres desses eventos, em 12 dias $90,1 \% \pm 4,8 \%(\mathrm{~N}=29)$, em 14 dias $83,6 \% \pm 6,3 \%(\mathrm{~N}=$ $25)$ e em 15 dias $80,2 \% \pm 6,9 \%(\mathrm{~N}=24)$.

Segundo Piepmeier et al. ${ }^{25}$ a bradicardia é uma alteração cardiovascular encontrada em $64 \%$ a $77 \%$ dos casos de TRM cervical. No trauma toracolombar, $13 \%$ a $35 \%$ evoluem para bradicardia. ${ }^{1,26}$ Em nossa amostra, dos pacientes com lesão nível alto, $11(35,5 \%)$ apresentaram hipotensão arterial e quatro $(12,9 \%)$, bradicardia. No entanto, dos pacientes com TRM nível baixo, dois $(11,1 \%)$ apresentaram hipotensão arterial $(p=0,06)$ e um $(5,6 \%)$ evoluiu com bradicardia $(p=0,41)$. Essa baixa prevalência de bradicardia deve-se ao número pequeno da amostra, não sendo possível saber a real percentagem dessa alteração cardiovascular nesse grupo de pacientes.

Os pacientes com lesão cervical completa (ASIA A e B) desenvolvem bradicardia em $77 \%$ a $100 \%$ dos casos e $68 \%$ desenvolvem hipotensão arterial. ${ }^{1,26}$ No entanto, Guly et al. ${ }^{23}$ relataram que a prevalência de hipotensão arterial em vítimas de lesões completas foi de 25,8\%. Dos pacientes com lesão cervical incompleta (ASIA C e D), $35 \%$ a $71 \%$ desenvolvem bradicardia e poucos desenvolvem hipotensão arterial. ${ }^{1,26}$ Este estudo revelou que, nos pacientes que tiveram TRM nível alto, o tipo de lesão completa esteve associado a maior frequência de bradicardia durante internação, sendo que $44,4 \%$ dos que tinham lesão completa apresentaram bradicardia. Também houve forte associação entre lesão completa e evolução para hipotensão arterial, sendo observado que $100 \%$ dos que tiveram lesão completa evoluíram para hipotensão arterial, contra apenas 14,3\% daqueles com lesão incompleta, concordando com dados da literatura.

Segundo Guly et al. ${ }^{23}$ a média da PA sistólica de 490 pacientes após TRM é de $120 \mathrm{mmHg}$, naqueles com lesões cervicais é de $110 \mathrm{mmHg}$ e toracolombar, 130 $\mathrm{mmHg}$. Em nossa amostra, a média da PA sistólica dos 49 pacientes na admissão foi maior $(123,6 \pm 17 \mathrm{mmHg})$ quando comparada à média durante a internação $(109,1$ $\pm 19,6 \mathrm{mmHg}$ ). Nas vítimas com lesões nível alto, essa média foi menor $(105,9 \pm 21,8 \mathrm{mmHg})$ quando comparada ao grupo TRM nível baixo $(114,7 \pm 13,9 \mathrm{mmHg})$.
Há necessidade de vasopressores em $35 \%$ dos pacientes com lesão cervical completa e poucos necessitam de vasopressores quando a lesão é cervical incompleta. ${ }^{27,28}$ Neste estudo, nove $(18,4 \%)$ pacientes necessitaram usar algum tipo de vasopressor, dos quais sete tinham TRM nível alto. Nesse sentido, houve forte associação entre lesão completa e uso de vasopressor, sendo que seis $(66,7 \%)$ dos que tiveram lesão completa usaram vasopressores contra três $(7,5 \%)$ daqueles com lesão incompleta $(\mathrm{P}<0,0001)$.

A mortalidade em pacientes com TRM que apresentam choque neurogênico é de $12,7 \%$, aumentando para $18,9 \%$ em casos de lesão cervical e diminuindo para 3,2\% quando torácica. ${ }^{24}$ Segundo Bilello et al. ${ }^{28}$ a mortalidade de pacientes com lesão cervical é de $12 \%$, sendo mais frequentes os óbitos quando o TRM é acima de C5. Para Grigorean et al., ${ }^{1}$ nos pacientes com lesões cervicais ocorre a parada cardíaca primária em $16 \%$ dos casos e as vítimas de TRM toracolombar raramente desenvolvem. Este trabalho revelou mortalidade de 20,4\% (10 pacientes), e a prevalência de parada cardíaca seguida do óbito foi maior naqueles com TRM nível alto, $80 \%(\mathrm{n}=8)$, mostrando concordância com dados da literatura. Esses dados revelam que, além do que é dito na literatura sobre a alta mortalidade em lesões cervicais, quando o TRM atinge as vértebras cervicais e torácicas altas (até T5) o prognóstico é pior, as DCV são mais frequentes e a mortalidade é maior.

A sobrevida global da amostra deste estudo foi menor $(77,3 \% \pm 7,5 \%$ em 20 dias) quando comparada à sobrevida relatada na literatura $(94 \%$ em pacientes que têm hospitalização rápida após o TRM). ${ }^{29}$ Foi observado também que a sobrevida em 20 dias dos que tiveram hipotensão arterial foi menor quando comparada aos que não tiveram hipotensão arterial, respectivamente de $59,8 \% \pm 14 \%$ versus $96,6 \% \pm 3,4 \%$. Esses dados revelam que a hipotensão arterial foi fator de risco independente para aumento da mortalidade. Em relação à sobrevida dos pacientes com TRM nível alto em 20 dias, observou-se que ela foi menor $(69,6 \%$ $\pm 9,8 \%)$ quando comparada à sobrevida dos pacientes com lesão nível baixo $(88,9 \% \pm 10,5 \%)$.

Dessa forma, pode-se perceber que a hipotensão arterial e bradicardia foram DCV frequentes em nossa amostra, tendo prevalência semelhante à da literatura médica. Porém, o TRM nível alto esteve mais associado a essas complicações, havendo péssimo prognóstico (menor sobrevida) desses pacientes neste estudo.

\section{Conclusões}

O TRM é uma patologia frequente em paciente adulto-jovem do gênero masculino. A principal causa 
é queda e a lesão cervical é a mais frequente. Bradicardia e hipotensão arterial são as DCV que mais surgem na fase aguda do TRM, estando associadas ao TRM nível alto e às lesões do tipo completa. O prognóstico das vítimas do TRM depende do nível e tipo da lesão, sendo a evolução para óbito frequente nas lesões nível alto e completa. A hipotensão arterial foi fator de risco independente para aumento da mortalidade, no entanto não foi possível concluir o mesmo da bradicardia pelo pequeno número de casos dessa alteração cardiovascular na amostra.

\section{Conflitos de interesse}

Os autores declaram não haver conflitos de interesse.

\section{Referências}

1. Grigorean VT, Sandu AM, Popescu M, lacobini MA, Stoian $R$, Neascu $C$, et al. Cardiac dysfunctions following spinal cord injury. J Med Life. 2009;2(2):133-45.

2. $\mathrm{Ho} \mathrm{CH}$, Wuermser LA, Priebe MM, Chiodo AE, Scelza WM, Kirshblum SC. Spinal cord injury medicine. 1. Epidemiology and classification. Arch Phys Med Rehabil. 2007;88(3 Suppl 1):S49-54.

3. Defino HLA. Trauma raquimedular. Medicina (Ribeirão Preto). 2000;32(4):388-99.

4. Brito LMO, Chein MBC, Marinho SC, Duarte TB. Avaliação epidemiológica dos pacientes vítimas de traumatismo raquimedular. Rev Col Bras Cirurg. 2001;38:305-9.

5. Greve JM. Traumatismos raquimedulares nos acidentes de trânsito e uso de equipamentos de segurança. Diag e Tratam. 1997;2(1):10-3.

6. Venturini DA, Decésaro M, Marcon SS. Changes and expectations experienced by individuals with spinal cord injury and their families. Rev Esc Enferm USP. 2007;41(4):589-96.

7. Furuland $\mathrm{H}$, Linde $\mathrm{T}$, Englund $\mathrm{A}$, Wikström B. Heart rate variability is decreased in chronic kidney disease but may improve with hemoglobin normalization. J Nephrol. 2008;21(1):45-52.

8. Wuermser LA, Ho CH, Chiodo AE, Priebe MM, Kirshblum SC, Scelza WM. Spinal cordinjury medicine. 2. Acute care management of traumatic and non traumatic injury. Arch Phys Med Rehabil. 2007;88(Suppl 3):S55-61.

9. Schottler J, Vogel L, Chafetz R, Mulcahey MJ. Patient and caregiver knowledge of autonomic dysreflexia among youth with spinal cord injury. Spinal Cord. 2009;47(9):681-6.

10. McMahon D, Tutt M, Cook AM. Pharmacological management of hemodynamic complications following spinal cord injury. Orthopedics. 2009;32(5):331.

11. Popa C, Popa F, Grigorean VT, Onose G, Sandu AM, Popescu M, et al. Vascular dysfunctions following spinal cord injury. J Med Life. 2010;3(3):275-85.

12. Scramin AP. Convivendo com a tetraplegia: da necessidade de cuidados à integralidade no cotidiano de homens com lesão medular cervical [dissertação]. Maringá: Universidade Estadual de Maringá; 2006.
13. Lenehan B, Street J, Kwon BK, Noonan V, Zhang H, Fisher $\mathrm{CG}$, et al. The epidemiology of traumatic spinal cord injury in British Columbia, Canada. Spine (Phila Pa 1976). 2012;37(4):321-9.

14. Ning GZ, Yu TQ, Feng SQ, Zhou XH, Ban DX, Liu Y, et al. Epidemiology of traumatic spinal cord injury in Tianjin, China. Spinal Cord. 2011;49(3):386-90.

15. Liu P, Yao Y, Liu MY, Fan WL, Chao R, Wang ZG, et al. Spinal trauma in mainland China from 2001 to 2007: an epidemiological study based on a nationwide database. Spine (Phila Pa 1976). 2012;37(15):1310-5.

16. Defino HLA, Zardo EA. Trauma raquimedular. In: Hebert S, Barros Filho TE, Xavier R, Pardini Junior AG. Ortopedia e traumatologia: princípios e prática. 4a. ed. Porto Alegre: Artmed; 2008. p. 1024-34.

17. Pickett GE, Campos-Benitez M, Keller JL, Duggal N. Epidemiology of traumatic spinal cord injury in Canada. Spine (Phila Pa 1976). 2006;31(7):799-805.

18. Hagen EM, Eide GE, Rekand T, Gilhus NE, Gronning M. A 50-year follow-up of the incidence of traumatic spinal cord injuries in Western Norway. Spinal Cord. 2010;48(4):313-8.

19. Andrade SM, Jorge MH. Characteristics of the victims of traffic accidents in a city of the south ern region of Brazil. Rev Saude Publica. 2000;34(2):149-56.

20. Slucky AV, Eismont FJ. Treatment of acute injury of the cervical spine. J Bone Joint Surg Am. 1994;76(12):1882-9.

21. Gonçalves $A M T$, Rosa $L N$, D'ângelo $C T$, Savordelli $C L$, Bonin GL, Squarcino IM. Aspectos epidemiológicos da lesão medular traumática na área de referência do Hospital Estadual Mário Covas. Arq Méd ABC. 2007;32:64-6.

22. Krassioukov AV, Karlsson AK, Wecht JM, Wuermser LA, Mathias CJ, Marino RJ. Assessment of autonomic dysfunction following spinal cord injury: rationale for additions to international standards for neurological assessment. J Rehabil Res Dev. 2007;44(1):103-112.

23. Guly HR, Bouamra O, Lecky FE. The incidence of neurogenic shock in patients with isolated spinal cord injury in the emergency department. Resuscitation. 2008;76(1):57-62.

24. Zipnick RI, Scalea TM, Trooskin SZ, Sclafani SJ, Emad B, Shah A, et al. Hemodynamic responses to penetrating spinal cord injuries. J Trauma.1993;35(4):578-82.

25. Piepmeier JM, Lehmann KB, Lane JG. Cardiovascular instability following acute cervical spinal cord trauma. Cent Nerv Syst Trauma. 1985;2(3):153-60.

26. Mckinley W, Garstang SV, Wieting JM, Talavera F, Foye $\mathrm{PM}$, Allen KL, et al. Cardiovascular concerns in spinal cord injury. eMedicine; 2006. Disponível em: http://emedicine. medscape.com/article/321771-overview

27. Lehmann KG, Lane JG, Piepmeier JM, Batsford WP. Cardiovascular abnormalities accompanying acute spinal cord injury in humans: incidence, time course and severity. J Am Coll Cardiol. 1987;10(1):46-52.

28. Bilello JF, Davis JW, Cunningham MA, Groom TF, Lemaster D, Sue LP. Cervical spinal cord injury and the need for cardiovascular intervention. Arch Surg. 2003;138(10):1127-9.

29. Gondim FAA, Thomas FP, Berman SA, Talavera F, Kirshner HS, Bembadis SR. Spinal cord trauma and related diseases clinical presentation. eMedicine; 2011. Disponível em: http://emedicine.medscape.com/article/321771-overview.

Endereço para correspondência

Liani Patrícia Andrade Santos

Rua Armando Barros, 81, Bairro Luiza

49045-080 - Aracaju, SE, Brasil

Email: liani.paty@gmail.com 